\title{
PESSOAS IDOSAS COM COVID-19 EM AMBIENTES DE CUIDADOS CRÍTICOS
}

Maria de Nazaré de Souza Ribeiro'

ORCID: 0000-0002-7641-1004

Cleisiane Xavier Diniz'

ORCID: 0000-0003-4689-6204

Matheus Souza Silva" ORCID: 0000-0001-8159-0687

Universidade do Estado do Amazonas, Escola Superior de Ciências da Saúde. Manaus, Amazonas, Brasil

"Hospital Universitário Dr. Miguel Riet Correa Jr. da Universidade Federal do Rio Grande. Rio Grande, Rio Grande do Sul, Brasil

Autor Correspondente: Maria de Nazaré de Souza Ribeiro E-mail: mnribeiro2@gmail.com

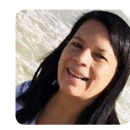

Como citar:

Ribeiro MNR, Diniz CX, Silva MS. Pessoas idosas com covid-19 em ambientes de cuidados críticos. In: Santana RF. Enfermagem gerontologica no cuidado do idoso em tempos da COVID 19. 2.ed.rev. Brasilia, DF: Editora ABEn; 2020. p. 49-54. (Serie Enfermagem e Pandemias, 1). https://doi.org/10.51234/aben.20.e01.c08

\section{INTRODUÇÃO}

A disseminação acelerada do Covid-19, também chamado de Sars-Cov-2 (Severe acute respiratory syndrome coronavirus 2), tornou-se uma grande ameaça à saúde pública e um desafio para o mundo. Estudos mostram que as internações e mortes por COVID-19 variam significativamente entre os diferentes subgrupos etários. Pessoas com 50 anos ou mais têm chance 2,6 vezes de progredir para um caso grave de Covid-19, sendo que os idosos estão entre os mais afetados. As taxas de mortalidade pela infecção aumentam com a idade e são as mais altas entre aqueles de 80 anos ${ }^{(1,2)}$.

Em uma metanálise realizada por Zhao et al. ${ }^{(1)}$, incluindo 30 estudos, totalizando 53.000 pacientes com COVID-19, identificou-se que idosos do sexo masculino e com presença de comorbidades, incluindo hipertensão, diabetes, câncer e doenças respiratórias, foram identificados como preditores da gravidade da doença e da mortalidade relacionada ao COVID-19, sugerindo que pacientes idosos com essas comorbidades subjacentes devam receber mais atenção e cuidado. Ainda segundo Zhao et al. ${ }^{(1)}$, doenças graves e mortes são mais prováveis de ocorrer entre aqueles com condições de saúde subjacentes, incluindo doenças respiratórias (como doença pulmonar obstrutiva crônica, asma), doenças cardiovasculares e diabetes. Assim, as estatísticas mostram que as admissões e mortes hospitalares de COVID-19 exibem um padrão etário distinto, onde até um quinto da população idosa infectada agrava e morre.

Na evolução da doença, as pessoas infectadas apresentam progressiva dificuldade respiratória, síndrome do desconforto respiratório agudo ou complicações com risco de vida, levando a uma necessidade de cuidados intensivos. Como em muitos casos os idosos já possuem doenças subjacentes e, portanto, costumam apresentar imunossupressão, os sintomas podem aparecer de forma atípica, com risco comum para o mau prognóstico para a Covid-19. Um estudo mostra que o maior número de 
pacientes que evoluíram da Síndrome de Angústia Respiratória Aguda (SRAG) parte das mortes eram em idosos, com elevação de bilirrubina, ureia, proteína $C$ reativa e $D$-dímero e, quase sempre sem apresentar febre $\geq 39^{\circ} \mathrm{C}(\mathrm{WU}, 2020)$. A Hipertensão e doenças cardiovasculares são os preditores clínicos mais relevantes para a morte causada pela COVID-19 e MERS-CoV, enquanto a doença renal crônica esteve para a SARS ${ }^{(1,3)}$.

Considerando que as intervenções terapêuticas a pacientes críticos, diferem entre pacientes idosos e não idosos, dada a vulnerabilidade caracterizada principalmente por sua condição funcional e presença de comorbidades, destaca-se, nesse sentido, a importância da participação efetiva da enfermagem nos cuidados prestados aos idosos com a COVID-19 em ambientes críticos. Pois, tradicionalmente, considera-se que a assistência à pacientes críticos/potencialmente críticos seja prestada em ambientes especializados, como as Unidades de Tratamento Intensivo (UTI) e Semi-Intensivo (UTSI).

\section{OBJETIVO}

Refletir sobre os desafios para a enfermagem nos cuidados aos pacientes idosos com a COVID-19 em ambientes de cuidados críticos.

\section{MÉTODO}

Essa reflexão, elaborada num cenário de poucas evidências científicas sobre a COVID-19, destacou-se as seguintes situações: manejo ventilatório no idoso com SRAG e COVID-19 em ambiente crítico; e manejo clínico da sepse associada à infecção por SARS-CoV-2 em pacientes idosos.

Refletir sobre este cuidado é necessário em virtude do grande contingente de população idosa que tem sido acometida pela doença e muitos que evoluem para a necessidade de cuidados intensivos de enfermagem. Ressalta-se que não será abordado nesta reflexão aspectos de decisões éticas que envolvem seleção individualizada de beneficiários relacionada à idade para o número insuficiente de vagas nos serviços de terapia intensiva. Ressalta-se que o cenário de poucas evidências sobre o controle, tratamento e prognósticos dos doentes de COVID-19 exige cautela na proposição de esquemas de cuidados pela enfermagem à pacientes idosos e um grande controle dos fatores de agravamento do problema. Assim, as indicações referidas nesse estudo, foram baseadas na literatura científica disponível para atendimento às pessoas idosas em ambientes críticos, com adaptação para atendimento à idosos com COVID-19, uma vez que não há literatura disponível que trata sobre o tema.

\section{RESULTADOS}

\section{MANEJO VENTILATÓRIO NO IDOSO COM SRAG E COVID-19 EM AMBIENTE CRÍTICO}

O envelhecimento causa alterações estruturais e funcionais no sistema respiratório podendo levar o idoso crítico a graves complicações, como por exemplo a calcificação das cartilagens costais ocasionando o aumento no gasto energético despendido na respiração. A complacência pulmonar e a área de superfície alveolar reduzem e levam ao aumento do volume residual pulmonar diminuindo a troca de gases, fazendo assim que o centro respiratório apresente sensibilidade reduzida, aumentando o consumo de oxigênio e também da produção de gás carbônico. Aumentando o risco de lesão pulmonar e Síndrome Respiratória Aguda Grave (SRAG), caracterizada pela alteração da membrana alvéolo-capilar, sendo causada por lesão de natureza inflamatória que determina a formação do edema que extravasa para o interior dos alvéolos, levando a insuficiência respiratória aguda ${ }^{(4,5)}$.

Os critérios para definição de SRAG são baseados em achados clínicos, gasométricos e radiológicos. Nos pacientes acometidos pelo COVID-19, a evolução do quadro gripal para SRAG ocorre de forma muito rápida. $\mathrm{E}$, como critério clínico tem-se o exame físico realizado no paciente, com sinais de taquipneia, esforço respiratório, bem como, a ausculta pulmonar com sons crepitantes finos inspiratórios difusos; no exame radiológico (raio X de tórax) há infiltrados bilaterais; e na gasometria arterial a relação $\mathrm{PaO}_{2} / \mathrm{FiO}_{2}<200^{(4,6)}$. 
Uma das alternativas mais eficazes no tratamento da SRAG é a colocação do paciente em posição prona a qual melhora a troca gasosa através da expansão da região dorsal dos pulmões, aumentando o volume expiratório pulmonar e melhorando a relação ventilação-perfusão. O benefício da posição prona está combinado com os seguintes mecanismos: alteração da insuflação pulmonar regional, a redistribuição da ventilação e a redistribuição da perfusão ${ }^{(4,6)}$.

Ainda não há um consenso no que tange o tempo de posição prona a qual o paciente deve ser submetido. As evidências estão mostrando a melhora da oxigenação durante as primeiras 2 horas do procedimento, com pequenos acréscimos nas 4 horas seguintes. Está indicado a coleta a cada hora de gasometria arterial para verificação dos valores de $\mathrm{PaO}_{2}$. Segundo o Ministério da Saúde, a indicação mínima de prona para os casos de COVID-19 é de 16 horas $^{(4)}$. A posição prona traz algumas complicações, e aos pacientes idosos merecem destaque: as lesões por pressão de olhos e pálpebras, lesões na boca e língua, lesões de pele (skin tears); extubação acidental(4,5).

Logo, destaca-se como cuidados de enfermagem para pacientes idosos com SRAG em posição prona e/ou supina: Cabeceira elevada em 30-45, pois esse posicionamento evita a aspiração pulmonar do refluxo gástrico; Avaliar a expansão e simetria torácica verificando se o paciente está ventilando bilateralmente; Realização de higiene oral utilizando solução antisséptica a cada 6 horas ou sempre que necessário a fim de evitar infecções; Otimizar a condição hemodinâmica antes da prona, observando o surgimento de hipotensão ou arritmias cardíacas; Atentar aos cateteres, conexões, sondas e drenos antes, durante e após a virada; Proteger locais de apoio ou de maior pressão: queixo, orelhas, região anterior do tórax, cristas ilíacas e joelhos através da utilização de coxins para posicionamento ideal evitando fricção e cisalhamento das proeminências ósseas; Alternar lado da cabeça a cada 2 a 4 horas enquanto estiver pronado com vistas a redução do edema facial e lesões do nervo periférico e cegueira, pela excessiva pressão sobre os olhos ${ }^{(4)}$.

Destaca-se ainda o forte impacto ao sistema respiratório devido as próprias alterações do envelhecimento como a falência respiratória por resposta deficiente à hipoxemia e hipercapnia; o risco aumentado para desequilíbrio da relação ventilação-perfusão; aumento da sensibilidade aos narcóticos; dificuldade de cicatrização de lesões como consequência da oxigenação diminuída; e risco aumentado de dependência de ventilação mecânica (4).

\section{MANEJO CLÍNICO DA SEPSE ASSOCIADA À INFECÇÃO POR SARS-COV-2 EM PACIENTES IDOSOS}

Em estudo realizado com 191 pacientes com COVID-19, na cidade chinesa de Wuhan, Zhou et al ${ }^{(7)}$, chegaram à conclusão que a idade avançada é um fator de risco para a Covid-19, com um índice de mortalidade mais elevada em idosos com média de idade de 69 anos. Em todos os pacientes, a complicação mais comum decorrente da COVID-19, foi a sepse (detectada em 100\% dos pacientes mortos e $42 \%$ dos sobreviventes), seguida de insuficiência respiratória ( $98 \%$ versus $36 \%$ ). A frequência de complicações como essas foi maior em pessoas que morreram do que as que tiveram alta ${ }^{(7)}$.

A sepse é uma síndrome ameaçadora à vida em decorrência da presença de resposta desregulada à infecção. Os termos mais utilizados para classificação da sepse, indicados pelo Instituto Latino Americano de Sepse são: Infecção sem disfunção, Sepse e Choque Séptico ${ }^{(8)}$.

Os protocolos clínicos gerenciados de sepse são uma ferramenta imprescindível, auxiliando os enfermeiros no alinhamento do atendimento ao paciente séptico, diminuindo desfechos negativos e proporcionando melhor efetividade no tratamento. Deve ser aberto para todos os pacientes com SUSPEITA de Sepse e Choque Séptico.

No entanto, é necessário observar as principais disfunções orgânicas que podem se fazer presentes como: hipotensão (PAS $<90 \mathrm{mmHg}$ ou PAM $<65 \mathrm{mmHg}$ ou queda de PA $>40 \mathrm{mmHg}$ ); rebaixamento do nível de consciência, agitação, delirium; relação $\mathrm{PaO} 2 / \mathrm{FiO} 2$ < 300 ou necessidade de $\mathrm{O} 2$ para manter $\mathrm{SpO} 2$ > 90\%; contagem de plaquetas $<100.000 / \mathrm{mm}^{3}$ ou redução de $50 \%$ no número de plaquetas em relação ao maior valor registrado nos últimos 3 dias; lactato acima do valor de referência; aumento significativo de bilirrubinas ( $>2 X$ o valor de referência); e oligúria $(\leq 0,5 \mathrm{~mL} / \mathrm{Kg} / \mathrm{h})$ ou elevação da creatinina $(>2 \mathrm{mg} / \mathrm{dL})^{(8)}$. 
Na presença de uma dessas disfunções orgânicas citadas, sem outra explicação plausível e com foco infeccioso presumível, o diagnóstico de sepse deve ser feito, e o pacote de tratamento iniciado, imediatamente após a identificação, seguindo as recomendações contidas e baseadas nas diretrizes da Campanha de Sobrevivência a Sepse (SSC), que visam o tratamento de pacientes adultos e idosos nas unidades de urgência e emergência, nas unidades de internação e nas unidades de terapia intensiva (UTIs).

Dois indicadores detectados na entrada dos pacientes idosos com COVID-19 nos hospitais, que podem ajudar a rastrear mais cedo casos potencialmente graves relacionado aos efeitos da COVID-19, é uma pontuação alta no índice de avaliação sequencial de falha de órgão (qSOFA score), uma avaliação comumente feita nas Unidades de Terapia Intensiva (UTIs). No escore qSOFA, exames e outras informações sobre o paciente são coletadas e compõem uma pontuação que indica uma menor ou maior inclinação à falha dos órgãos devido à sepse. É necessário avaliar 3 componentes: rebaixamento de nível de consciência, frequência respiratória $\geq \mathbf{2 2}$ ipm e pressão arterial sistólica abaixo de $100 \mathbf{m m H g}$. Sendo que nesse escore, identificaria entre pacientes fora da UTI aqueles com maior risco de óbito ${ }^{(6,8)}$.

Outro indicador é um valor de dímero-D alto no sangue (maior que $1 \mu \mathrm{g} / \mathrm{mL}$ ), fragmentos de proteína relacionados ao processo de coagulação. Casos de sepse e choque séptico normalmente vêm associados à chamada coagulação intravascular disseminada e que, por si só, eleva o Dímero-D. O estudo de Zhou et $a l^{(7)}$, indicou que casos mais graves de COVID-19 com sepse estavam relacionados a uma menor contagem de linfócitos; níveis elevados de Interleucina 6; e aumento das concentrações de Troponina I de alta sensibilidade. Níveis aumentados dessa troponina foram detectados em mais da metade dos pacientes que morreram.

Após triagem de pacientes com SUSPEITA de sepse, realizada pelo enfermeiro, a equipe médica decide se haverá o seguimento do protocolo. Para isso alguns fatores são levados em consideração ${ }^{(8)}$ :

1. Em pacientes idosos com qualquer das disfunções clínicas utilizadas na triagem (hipotensão, rebaixamento de consciência, dispneia ou dessaturação e, eventualmente, oligúria), segue-se o protocolo, com as medidas do pacote de 1 hora, e reavaliação ao longo das 6 primeiras horas.

2. Em pacientes idosos com disfunção clínica aparente, mas com quadro clínico sugestivo de outros processos infecciosos atípicos (no contexto da sepse), pode-se optar por seguir fluxo específico de atendimento que leve em consideração peculiaridades do atendimento a esses pacientes.

3. Em pacientes idosos sem disfunção clínica aparente, deve-se levar em conta o quadro clinico, não sendo adequado o seguimento do protocolo em pacientes com quadros típicos de infecções de via aérea alta ou amigdalites, por exemplo, que podem gerar SRIS (Systemic Inflammatory Response Syndrome), mas tem baixa probabilidade de se tratar de casos de sepse.

4. Em pacientes idosos para os quais já exista definição de cuidados de fim de vida, o protocolo deve ser descontinuado, e o paciente deve receber tratamento pertinente a sua situação clínica, incluindo eventualmente alguns dos componentes do pacote de tratamento.

Os pacientes são classificados e identificados da seguinte forma: paciente com infecção sem disfunção com seguimento do protocolo; sepse ou choque; afastado sepse/choque; paciente sob cuidados de fim de vida. Após essa identificação segue-se com: registro do diagnóstico no prontuário; prioridade no atendimento aos pacientes com protocolos de sepse abertos, com o objetivo de otimizar a coleta de exames, o início de antibioticoterapia e a ressuscitação hemodinâmica; realização da anamnese e do exame físico, com atenção especial aos sinais clínicos de disfunção orgânica; alocação em leitos de terapia intensiva de pacientes com disfunção orgânica grave e ou choque, a fim de garantir o suporte clínico necessário ${ }^{(8)}$.

Caso não seja possível a alocação em UTI, independente do setor em que o mesmo se encontre, a assistência de enfermagem deve ser garantida de maneira integral e a ficha do protocolo de sepse deve acompanhar o paciente durante todo o atendimento para facilitar a comunicação entre as equipes de diferentes turnos ou setores. 
Quanto ao Choque Séptico nos idosos, a suspeita, reconhecimento ou confirmação podem ser avaliadas pela presença de infecção e os vasopressores forem necessários para manter a pressão arterial média (PAM) $\geq 65 \mathrm{mmHg}$, aumento de lactato; estado mental alterado; hipertermia ou hipotermia; taquicardia ou bradicardia; taquipneia, recarga capilar prolongada (>2 s) ou vasodilatação quente com pulsos delimitadores; pele manchada ou erupção petequial ou purpúrica; oligúria. E o reconhecimento precoce e o uso do protocolo do tratamento de Sepse inclui na primeira hora a terapia antimicrobiana, e início de carga de fluidos mais vasopressores para a hipotensão(6).

Na ressuscitação do choque séptico (PAS $<90 \mathrm{mmHg}$, PAM $65 \mathrm{mmhg}$ ) é indicado o uso de vasopressores (drogas vasoativas) para pacientes que permaneçam com pressão arterial média (PAM) abaixo de $65 \mathrm{mmhg}$ (após a infusão de volume inicial), sendo a noradrenalina a droga de primeira escolha. Não se deve tolerar pressões abaixo de $65 \mathrm{mmHg}$ por períodos superiores a 30-40 minutos. Por isso, o vasopressor deve ser iniciado dentro da primeira hora nos pacientes em que ele está indicado. E em casos de hipotensão severa (ameaçadora à vida), deve-se iniciar o vasopressor mesmo antes ou durante a reposição volêmica. Se sinais de hipoperfusão persistirem, considerar uso de droga inotrópica ${ }^{(8)}$.

Caso os cateteres venosos centrais não estejam disponíveis, os vasopressores podem ser administrados por meio de um IV periférico, mas é necessário uma veia de grande calibre e monitorar de perto os sinais de extravasamento e necrose tecidual no local da inserção do dispositivo intravenoso. Caso ocorra extravasamento para a infusão, os vasopressores também podem ser administrados através de agulhas intraósseas.

É recomendado a utilização de dispositivos invasivos (cânula orotraqueal, cateter venoso central, pressão arterial invasiva e cateter vesical de demora) pelo menor tempo possível afim de se evitar complicações mecânicas e infecciosas, e que as unidades sigam as diretrizes para prevenção de infecções relacionadas a estes dispositivos (pneumonia associada a ventilação mecânica, infecção de corrente sanguínea associada a cateter venoso central e infecção do trato urinário relacionado a cateter vesical de demora) $)^{(6,8)}$.

Deve-se ainda levar em consideração os demais diagnósticos diferenciais pertinentes e o adequado manejo clínico. $O$ atendimento ao paciente idoso séptico nas primeiras 24 horas é de extrema importância para o desfecho favorável. E outras ações são necessárias para o sucesso de sobrevida hospitalar e reabilitação após a alta, com estabelecimento de uma linha de cuidado adequada, desde o momento da internação ou do diagnóstico de sepse até o momento da alta.

A integração de toda equipe é importante, abrangendo a Nutrição adequada; fisioterapia respiratória e motora, focada em mobilização precoce; o atendimento fonoaudiológico para recuperação de fonação e deglutição; o apoio do psicológico para minimizar os riscos de desenvolvimento de estresse; a odontologia na identificação e controle do foco infeccioso, bem como na prevenção de infecções respiratórias; a farmácia que possibilita a adequação da prescrição médica; o serviço social para a reinserção do paciente na sociedade e o seguimento do mesmo no sistema de saúde. Porém, a enfermagem é de importância fundamental e essencial para a integração de todos esses aspectos da assistência, na recuperação funcional do paciente idoso, desde o momento da sua admissão na unidade de cuidados críticos, até a alta hospitalar.

\section{CONCLUSÃO}

Face à epidemia da COVID-19 a demanda por serviços de saúde tem aumentado, levando uma parcela significativa de pacientes críticos/potencialmente críticos a ocuparem outros espaços assistenciais, onde adultos e idosos acabam por receber o mesmo tipo de atenção, algumas vezes até negligenciados em função da gravidade dos casos e do número reduzido de profissionais que prestam cuidados. Com isso, reforça-se o princípio de que o Enfermeiro deve assistir o paciente idoso, de forma que este receba o nível apropriado de assistência, independentemente de onde esteja alocado. As ações da enfermagem, na medida do possível, devem ser realizadas de modo holístico, integral, com compromisso ético, revestido de atitudes e respeito com os envolvidos, exigindo dos enfermeiros e técnicos de enfermagem, competência técnica-científica e humanística que direcione os cuidados aos idosos com a COVID-19 em ambientes de cuidados críticos. 


\section{AGRADECIMENTO}

\section{Ao Departamento Científico de Enfermagem Gerontológica da ABEn Nacional.}

\section{REFERÊNCIAS}

1. Zhao X, Zhang B, Li P, Ma C et al. Incidence, clinical characteristics and prognostic factor of patients with COVID-19: a systematic review and meta-analysis. COVID-19SARS-CoV-2 preprints from medRxiv and bioRxiv, 2020. doi: https://doi.org/10.1101/2020.03.17 20037572

2. Kulu H, Dorey P. The Contribution of Age Structure to the Number of Deaths from COVID-19in the UK by Geographical Units. COVID-19SARS-CoV-2 preprints from medRxiv and bioRxiv, 2020. doi: https://doi.org/10.1101/2020.04.16.20067991

3. Wu C, Chen X, Cai Y, Xia J et al. Risk Factors Associated With Acute Respiratory Distress Syndrome and Death in Patients With Coronavirus Disease 2019 Pneumonia in Wuhan, China. JAMA Intern Med. Published online March 13, 2020. doi:10.1001/jamainternmed.2020.0994

4. Viana RA.P, Torre M. Enfermagem em terapia intensiva: práticas integrativas. Barueri, SP: Manole, 2017.

5. Knobel E. Terapia Intensiva: enfermagem. São Paulo: Atheneu. 2010.

6. Brasil. Ministério da Saúde. Secretaria de Atenção Especializada à Saúde. Departamento de Atenção Hospitalar, Domiciliar e de Urgência. Protocolo de manejo clínico da COVID-19 na Atenção Especializada [recurso eletrônico] / Ministério da Saúde, Secretaria de Atenção Especializada à Saúde, Departamento de Atenção Hospitalar, Domiciliar e de Urgência. - 1. ed. rev. Brasília: Ministério da Saúde, 48 p. [Internet]. 2020 [cited 2020 Apr 22]; Available from: https://portalarquivos.saude.gov.br/ images/pdf/2020/April/14/Protocolo-de-Manejo-Cl--nico-para-o-Covid-19.pdf

7. Zhou F, Yu T, Du R, et al. Clinical course and risk factors for mortality of adult inpatients with COVID-19in Wuhan, China: a retrospective cohort study. Lancet 2020. Published online March 9. https://doi.org/10.1016/ S0140-6736(20)30566-3.

8. Instituto Latino Americano de Sepse (ILAS). Implementação de protocolo gerenciado de sepse protocolo clínico Atendimento ao paciente adulto com sepse / choque séptico. [Internet]. 2018 [cited 2020 Apr 22]; Available from: https:// www.ilas.org.br/assets/arquivos/ferramentas/ protocolo-de-tratamento.pdf. 